\title{
Gilles Dussault
}

Professeur agrégé, Département des relations industrielles, Université Laval chercheur au GRIS, Groupe de recherche interdisciplinaire en santé, Université de Montréal

(1994)

\section{"Les producteurs de services sociosanitaires”}

Un document produit en version numérique par Jean-Marie Tremblay, bénévole, Professeur sociologie au Cégep de Chicoutimi Courriel: jean-marie tremblay@uqac.ca

Dans le cadre de "Les classiques des sciences sociales"

Site web: http://classiques.uqac.ca/

Une bibliothèque fondée et dirigée par Jean-Marie Tremblay, sociologue

Une collection développée en collaboration avec la Bibliothèque Paul-Émile-Boulet de l'Université du Québec à Chicoutimi

Site web: http://bibliotheque.uqac.ca/ 


\section{Politique d'utilisation de la bibliothèque des Classiques}

Toute reproduction et rediffusion de nos fichiers est interdite, même avec la mention de leur provenance, sans l'autorisation formelle, écrite, du fondateur des Classiques des sciences sociales, Jean-Marie Tremblay, sociologue.

Les fichiers des Classiques des sciences sociales ne peuvent sans autorisation formelle:

- être hébergés (en fichier ou page web, en totalité ou en partie) sur un serveur autre que celui des Classiques.

- servir de base de travail à un autre fichier modifié ensuite par tout autre moyen (couleur, police, mise en page, extraits, support, etc...),

Les fichiers (.html, .doc, .pdf., .rtf, .jpg, .gif) disponibles sur le site Les Classiques des sciences sociales sont la propriété des Classiques des sciences sociales, un organisme à but non lucratif composé exclusivement de bénévoles.

Ils sont disponibles pour une utilisation intellectuelle et personnelle et, en aucun cas, commerciale. Toute utilisation à des fins commerciales des fichiers sur ce site est strictement interdite et toute rediffusion est également strictement interdite.

L'accès à notre travail est libre et gratuit à tous les utilisateurs. C'est notre mission.

Jean-Marie Tremblay, sociologue

Fondateur et Président-directeur général, LES CLASSIQUES DES SCIENCES SOCIALES. 
Cette édition électronique a été réalisée Jean-Marie Tremblay, bénévole, professeur de soins infirmiers retraitée de l'enseignement au Cégep de Chicoutimi

Courriel: jean-marie_tremblay@uqac.ca

à partir du texte de :

Gilles Dussault, “Les producteurs de services sociosanitaires”. Un article publié dans l'ouvrage sous la direction de Vincent Lemieux, Pierre Bergeron, Clermont Bégin et Gérard Bélanger, Le système de santé au Québec. Organisations, acteurs et enjeux. Chapitre 9, pp. 193-212. Québec : Les Presses de l'Université Laval, 1994, 370 pp.

M. Dussault était professeur agrégé au Département des relations industrielles de l’Université Laval. Maintenant chercheur dans le GRIS, le Groupe de recherche interdisciplinaire en santé, de l’Université de Montréal.

[Autorisation formelle de l'auteur accordée le 15 mai 2006 de diffuser cet article dans Les Classiques des sciences sociales.]

Courriel : gilles.dussault@umontreal.ca

GRIS : http://www.gris.umontreal.ca/rappshow.asp?idgroup=3\&numgrp=0\&chapX=t

Polices de caractères utilisée :

Pour le texte: Times New Roman, 14 points.

Pour les citations : Times New Roman 12 points.

Pour les notes de bas de page : Times New Roman, 12 points.

Édition électronique réalisée avec le traitement de textes Microsoft Word 2004 pour Macintosh.

Mise en page sur papier format : LETTRE (US letter), 8.5'’ x 11'’)

Édition numérique réalisée le 2 février 2008 à Chicoutimi, Ville de Saguenay, province de Québec, Canada.

\section{G Fait avec}




\section{Gilles Dussault}

Professeur agrégé, Département des relations industrielles, Université Laval chercheur dans le GRIS, le Groupe de recherche interdisciplinaire en santé, de l’Université de Montréal

\section{"Les producteurs de services sociosanitaires.”}

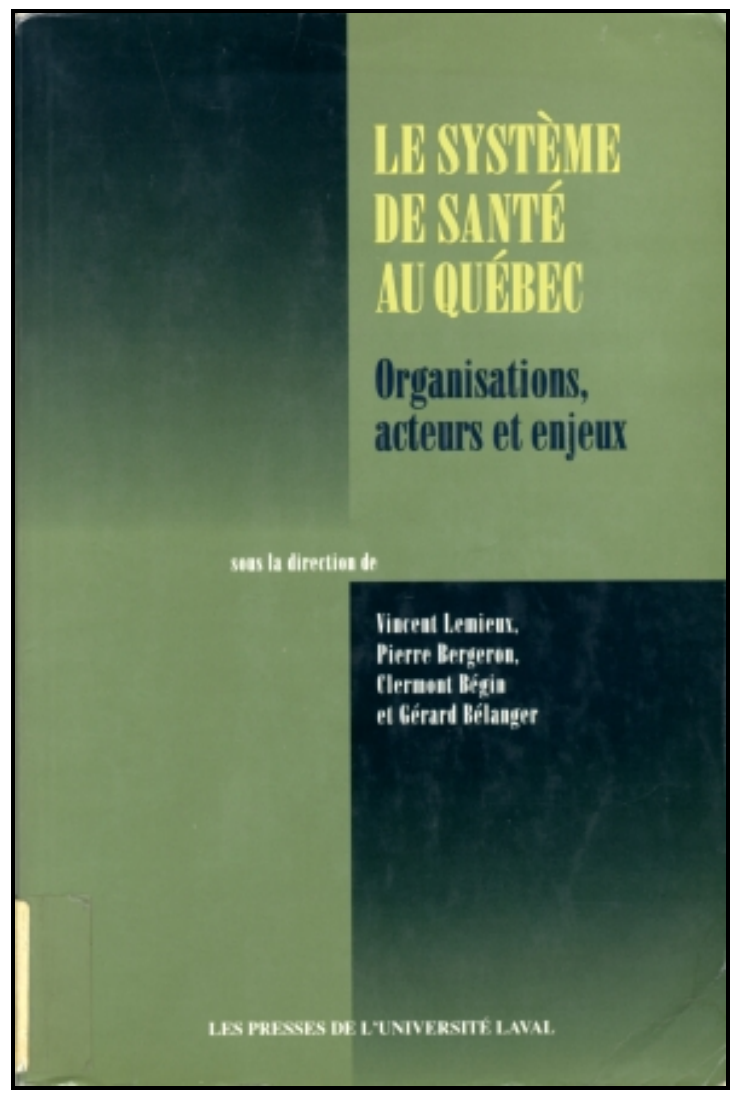

Un article publié dans l'ouvrage sous la direction de Vincent Lemieux, Pierre Bergeron, Clermont Bégin et Gérard Bélanger, Le système de santé au Québec. Organisations, acteurs et enjeux. Chapitre 9, pp. 193-212. Québec : Les Presses de l'Université Laval, 1994, 370 pp. 


\section{Table des matières}

$\underline{\text { Introduction }}$

Les effectifs du réseau des services sociosanitaires (1978-1991)

Les effectifs des corporations professionnelles (1978-1992)

Les producteurs non reconnus

Tendances de l'évolution des effectifs sociosanitaires

$\underline{\text { Conclusion }}$

$\underline{\text { Références }}$

Tableau 1. Effectifs, par catégorie de personnel, en équivalents en temps plein (ETP) Services de santé et services sociaux, Province de Québec, 1978, 1984, 1991

Tableau 2. Personnel clinique, en équivalents en temps plein (ETP)Services de santé et services sociaux, Province de Québec, 1980, 1984, 1991

Tableau 3. Nombre de postes, selon le statut d'emploi, personnel clinique des services de santé et des services sociaux, Province de Québec, 1980, 1984, 1991

Tableau 4. Effectifs des corporations professionnelles du secteur de la santé et des services sociaux, Province de Québec, 1978,1984,1992

Tableau 5. Données sur les effectifs médicaux au Québec, 1972, 1978, 1984, 1990

Tableau 6. Âge moyen et proportion de médecins âgés de 60 ans et plus selon la spécialité, au Québec, 1990 
Gilles Dussault,

“Les producteurs de services sociosanitaires".

Un article publié dans l'ouvrage sous la direction de Vincent Lemieux, Pierre Bergeron, Clermont Bégin et Gérard Bélanger, Le système de santé au Québec. Organisations, acteurs et enjeux. Chapitre 9, pp. 193-212. Québec : Les Presses de l'Université Laval, 1994, 370 pp.

\section{Introduction}

$\underline{\text { Retour à la table des matières }}$

La production de soins et de services de santé consomme une part importante de notre richesse individuelle et collective (autour de 9,5 du produit intérieur brut du Québec en 1992). Ces sommes sont consacrées à l'achat de services d'experts qui affirment posséder des connaissances et des compétences les rendant capables de répondre aux besoins de santé des individus. La grande majorité de ces experts invoquent les fondements scientifiques de leur savoir pour légitimer leur statut de "producteur » de services de santé. Cette légitimité leur est d'ailleurs reconnue de diverses façons : par la loi, pour ceux qui sont reconnus par le Code des professions (L.R.Q., c. C-26) qui va jusqu'à leur accorder le monopole d'exercice de certains types d'activités ; de facto, par les organisations de soins qui les embauchent ou par l'État qui subventionne leur formation dans les établissements d'enseignement reconnus. On trouve dans ces catégories, au Québec, 22 " professions » auxquelles on reconnaît des privilèges d'utilisation de titres ${ }^{1}$ et d'exercice exclusif ${ }^{2}$, et une cinquantaine d'autres occupa-

1 Diététistes ; ergothérapeutes ; hygiénistes dentaires ; infirmières et infirmiers auxiliaires ; inhalothérapeutes ; orthophonistes et audiologistes ; physiothéra- 
tions, dont les membres exercent dans les services de santé et les services sociaux.

La production n'est toutefois pas limitée aux services de ces " producteurs reconnus ». Il y a toujours eu d'autres producteurs, dont le savoir avait une base empirique ou autre que scientifique (ésotérique, révélée, etc.), qui offraient leurs services en marge du système de soins officiel. Ce sont les rebouteurs, les guérisseurs, détenteurs d'un " don », et bien sûr les charlatans de tout acabit. On doit aussi inclure, dans cette catégorie de producteurs non reconnus, sans toutefois les assimiler aux premiers, ceux qui se réclament d'une autre rationalité que celle qui a dominé nos représentations de la santé et de la maladie sous l'influence de la médecine allopathique. Ce sont ces " experts » qui définissent leurs services tantôt comme alternatifs tantôt comme complémentaires à la médecine orthodoxe ; les plus connus et les plus consultés sont les acupuncteurs, les homéopathes, les naturopathes, les herboristes et plusieurs variétés de psychothérapeutes. Leur nombre est difficile à établir puisqu'ils pratiquent sans reconnaissance légale et sont donc potentiellement sujets à des poursuites ; on s'accorde toutefois pour dire qu'à partir du début des années 1970, ils ont crû en effectif de façon soutenue (Dussault et al., 1988 ; Rousseau et al., 1991). Avec les producteurs reconnus, ils forment ce que nous pourrions appeler le secteur formel de la production de soins.

La production informelle, qui constitue la face plus ou moins cachée du système de soins, représente probablement la part la plus importante, en quantité, des soins et services consommés. On y trouve les services produits par le réseau primaire de soutien, formé des parents, des amis, de l'entourage immédiat, et par le réseau secondaire, formé des bénévoles des groupes communautaires, des associations

peutes ; psychologues ; techniciens et techniciennes dentaires ; technologistes médicaux ; travailleurs sociaux.

2 Audioprothésistes ; chiropraticiens, dentistes ; denturologistes ; infirmières et infirmiers ; médecins ; opticiens d'ordonnances ; optométristes ; pharmaciens ; podiatres; techniciens en radiologie. 
d'entraide. L'ampleur de cette production, de même que son efficacité, sont mal connues. On commence à peine aussi à essayer de mieux harmoniser cette production informelle à l'autre plus formelle. En fait, le secteur informel est particulièrement valorisé, depuis deux décennies surtout, étant vu comme pouvant compenser « les failles du système » de soins officiel (Dumais et Lévesque, 1986) et comme favorisant la prise en charge individuelle des problèmes de santé et la réduction de la dépendance à l'endroit des experts, qui sont deux valeurs devenues importantes dans notre société.

Dans le présent chapitre, nous limiterons la discussion au secteur formel de la production sanitaire. Nous en décrirons l'évolution récente, à partir des données disponibles, qui ont malheureusement de nombreuses limites, et nous dégagerons les principaux enjeux, que soulèvent les tendances observées, pour l'avenir de la production des services sociosanitaires et pour la santé et la qualité de vie de la population.

Plusieurs raisons devraient nous inciter à mieux connaître les caractéristiques des producteurs de services de santé. C'est le bassin de producteurs (nombre total d'individus), sa composition ${ }^{3}$, son évolution qui déterminent la capacité théorique ou potentielle de production. La production réelle dépend du comportement des producteurs et de leurs décisions quant à leur lieu d'installation, au nombre d'heures travaillées, au style de pratique. En connaissant mieux les facteurs qui font varier le bassin de producteurs et les comportements de ceux-ci, on peut mieux prévoir, et éventuellement influencer l'offre de services.

Dans la mesure où on adhère à l'objectif d'offrir des services qui sont congruents aux besoins, il importe d'abord de connaître la capacité productive, dans ses dimensions autant qualitatives que quantitatives ; ensuite il faut pouvoir l'ajuster aux besoins, par exemple en mo-

3 Par âge, par sexe, par occupation. 
difiant le bassin (augmenter/diminuer le nombre de producteurs en ajustant le nombre de places de formation ou d'immigrants), en influençant les processus de formation pour agir sur les compétences et les attitudes.

Dans les faits, cela reste un idéal difficilement atteint. D'abord, la connaissance des besoins en services est limitée par l'absence de données épidémiologiques ${ }^{4}$ et l'absence de consensus sur ce qu'est un « besoin ». Les producteurs, les utilisateurs, l'État, les organisations qui assument la charge financière des services, ont des visions différentes de ce qu'est un "besoin ». On peut illustrer cette affirmation avec l'exemple du débat sur l'opportunité d'offrir le service de sagesfemmes (Bardy, 1988), qui dure depuis le milieu des années 1970 et qui ne semble pas près d'une solution consensuelle. Ensuite, il n'est pas facile d'agir sur l'offre de services. La division et l'organisation du travail sanitaire sont fortement réglementées (lois professionnelles, contrats de travail), et il est difficile, politiquement, de les modifier radicalement. Les producteurs forment des groupes d'intérêt qui se sont donné les moyens de résister aux pressions de ceux qui cherchent à influencer leurs comportements, leur mode de pratique. Par exemple, les médecins et les chirurgiens-dentistes ont résisté avec succès aux tentatives de l'État d'atténuer les effets indésirables de leur monopole d'exercice en leur imposant une obligation de délégation (Dussault et al., 1988). Il en va de même pour la modification des contenus et des processus de formation qui dépendent essentiellement d'établissements d'enseignement autonomes ; leur capacité, sinon leur volonté, de s'ajuster rapidement à des besoins changeants n'est pas toujours aussi grande que l'État, les producteurs ou les utilisateurs de services de santé pourraient le souhaiter.

4 La première grande enquête de santé québécoise a été menée en 1987 (Émond et Guyon, 1988). Elle vient d'être reprise en 1992. Les autres données disponibles décrivent la mortalité, la morbidité diagnostiquée et l'utilisation des services : ces informations ne donnent qu'une image partielle des besoins, soit la partie qui donne lieu à des contacts avec le système de soins officiel. 
L'action sur l'offre de services est aussi limitée par notre ignorance relative de ses caractéristiques. On connaît bien les services couverts par le régime d'assurance-maladie du fait que les professionnels sont rémunérés à l'acte ; en présumant qu'ils réclament un paiement pour tous les actes exécutés et que ceux-ci ont été vraiment exécutés, on peut dire qu'on a une image exacte de la production de la plupart des services médicaux, de certains services dentaires et optométriques. Quant à la production non assurée, elle est plus mal connue : il faut se fier à quelques enquêtes, peu nombreuses, au cours desquelles on a essayé de mesurer et, plus rarement, de qualifier la production de services. Les services fournis par les producteurs non reconnus sont particulièrement mal connus. De plus, on ne connaît pas encore tous les facteurs qui influencent la productivité, ni ceux qui influencent les décisions des producteurs. On a observé des variations de comportement selon l'âge, le sexe, le mode de pratique, le lieu de pratique, les ressources disponibles, la formation reçue, mais il reste difficile d'isoler l'effet particulier de l'une ou l'autre de ces variables (Eisenberg, 1985).

Les objectifs de ce chapitre doivent être vus comme plutôt modestes, eu égard à ce qu'il faudrait idéalement savoir sur les producteurs de services. Les données disponibles et leurs limites inhérentes ${ }^{5}$ obli-

5 Les données disponibles sur la main-d'œuvre du secteur des services sociosanitaires proviennent de sources multiples qui ne fournissent que des informations partielles sur le profil socioprofessionnel des personnes qui produisent des services. Il est impossible actuellement de tracer un portrait détaillé du bassin de producteurs et même dans certains cas d'en évaluer la taille exacte en nombre d'individus. Ainsi, la source principale d'informations sur les employés du réseau public, les statistiques de la Direction générale des relations de travail (DGRT) du ministère de la Santé et des Services sociaux, fournit les données en nombre de postes. Les données sont tirées des fichiers de paie des établissements du réseau (elles englobent la quasi-totalité des établissements Publics et autour de 95\% des établissements privés conventionnés). Elles concernent les caractéristiques de la main-d'œuvre qui intéressent la gestion des conventions collectives (statut d'emploi, nombre d'heures travaillées, absences, etc.) ; il n'y a pas de données qui permettent de raffiner l'estimé de la production de services et de planifier les besoins en effectifs à partir de bases 
gent à nous en tenir à un aperçu général de l'évolution des effectifs sanitaires. Dans un premier temps, nous examinerons l'évolution des effectifs du réseau des services sociosanitaires, en concentrant l'attention sur le personnel clinique. Une deuxième section traitera des professionnels reconnus par le Code des professions. À l'exception des médecins, il est impossible de savoir quelle est la disponibilité réelle de ces professionnels : celle-ci dépend du nombre d'heures travaillées, de la proportion d'heures consacrées aux services comme tels, du type de services offerts, des modalités d'organisation du travail, de la productivité. Malgré les limites des informations disponibles, nous dégagerons les tendances de l'évolution des effectifs sanitaires et nous discuterons des questions qu'elles soulèvent.

plus solides. Par exemple, il n'y a pas d'information sur les tâches ellesmêmes, ni sur la formation ou les expériences du personnel. Compte tenu de leur origine et de l'usage premier auquel elles sont destinées, les données de la DGRT peuvent être considérées comme très fiables. Quant aux producteurs qui offrent leurs services sur le marché privé, les informations à leur sujet sont encore plus limitées. Les sources principales sont les corporations professionnelles, au nombre de 22 dans le secteur sociosanitaire. Pour les producteurs non regroupés en corporation, il n'y a souvent pas d'information du tout. Parmi les corporations, celles dites de " titre réservé » ne regroupent pas nécessairement l'ensemble des personnes exerçant l'activité qu'elles réglementent. Il en résulte que les données qu'elles produisent sont forcément partielles, à un degré qu'il n'est pas toujours possible d'estimer. Pour les corporations « d'exercice exclusif », les données sont complètes puisque le droit d'exercer est lié à l'inscription à la corporation. Toutefois, la majorité de ces corporations ne fournit que les informations socio-démographiques de base (âge, sexe) ; peu de données sont recueillies sur les activités des membres de sorte qu'il est difficile d'estimer la production réelle de services. La Corporation des médecins fait exception, comme on le verra plus loin dans le texte. 


\section{Les effectifs du réseau des services sociosanitaires (1978-1991)}

\section{$\underline{\text { Retour à la table des matières }}$}

Le réseau des services sociosanitaires est de loin le plus gros employeur au Québec, avec plus de 200000 personnes, dont les trois quarts sont des femmes, qui y travaillent. Les dépenses occasionnées par l'emploi de ces personnes représentent plus de 80\% des dépenses gouvernementales dans le secteur «santé et adaptation sociale ». En 1991-1992, la part des dépenses de ce secteur dans le budget de l'État était de 31,3\%, dont 6,6\% consacrée au régime d'assurance-maladie et 14,8\% aux hôpitaux de courte durée (RAMQ, 1992, p. 24).

Il est quasi impossible de connaître le nombre exact de personnes à l'emploi du réseau des affaires sociales. On connaît le nombre de postes et leur équivalent en temps plein, mais on ne peut savoir combien de personnes travaillent dans le réseau. De nombreux postes sont à temps partiel ou sont occupés pour une période limitée et un même individu peut être compté plus d'une fois si, au cours d'une année, il occupe plus d'un poste. L'information la plus significative est donnée par les statistiques qui ramènent les emplois à leur équivalent en temps plein (ETP) ${ }^{6}$.

Le tableau 1 montre l'évolution du nombre total de postes par catégorie d'emploi, selon les données du ministère de la Santé et des Services sociaux. Alors que la population du Québec a augmenté de 8,6\% de 1978 à 1991 7, les effectifs du réseau ont augmenté du double, soit

6 Un poste équivalent en temps plein est défini comme correspondant au temps travaillé par un employé à temps complet régulier sur une période d'un an, ce qui équivalait à 1826,3 heures en 1991.

7 La population du Québec était de 6302400 en 1978, de 6544277 en 1984 et estimée à 6845000 en 1991. 
de 17,9\%. L'essentiel de cette croissance (14,7\%) est survenue après 1984, et est attribuable à la croissance du personnel clinique (professionnels, infirmières, techniciens, assistants-techniciens). Leur nombre est passé de 83162 à 109123 (+31,1\%) alors que celui des autres personnels (employés de bureau, ouvriers, cadres) baissait de 60901 à 60231 (-1,1\%). Le nombre d'employés par cadre est passé de 10,86 à 12,30 au cours de la période. Ces données tendent à contredire les discours sur la diminution de la disponibilité des services cliniques et sur la croissance de la taille de l'encadrement au détriment des services aux usagers.

\section{Tableau 1}

Effectifs, par catégorie de personnel, en équivalents en temps plein (ETP) Services de santé et services sociaux, Province de Québec, 1978, 1984, 1991

Retour à la table des matières

\begin{tabular}{lrrrrr}
\hline & \multicolumn{3}{c}{ Nombre de postes ETP } & \multicolumn{2}{c}{$\begin{array}{c}\text { Accroissement } \\
1978=100\end{array}$} \\
\cline { 2 - 6 } & \multicolumn{1}{c}{1978} & 1984 & 1991 & 1984 & 1991 \\
\hline Professionnels & 5425 & 7440 & 11161 & 137 & 206 \\
Infirmières & 24323 & 27302 & 34811 & 112 & 143 \\
Techniciens & 14662 & 16912 & 21457 & 116 & 147 \\
Assistants-techniciens & 38792 & 38533 & 41694 & 99 & 107 \\
Employés de bureau & 19236 & 18669 & 21066 & 97 & 110 \\
Ouvriers & 29508 & 26643 & 26391 & 80 & 89 \\
Cadres & 12157 & 12531 & 12774 & 103 & 105 \\
TOTAL & 144103 & 148030 & 169354 & 103 & 118 \\
\hline
\end{tabular}

Sources : G. Dussault et al. (1987), La main-d'oeuvre socio-sanitaire au Québec, Québec, Commission d'enquête sur les services de santé et les services sociaux, p. 401-402. Ministère de la Santé et des Services sociaux, Province de Québec (1992), Statistiques sur le personnel de la santé et des services sociaux, 19901991, Québec, p. 308-312. 
Le tableau 2 limite l'information au personnel clinique, tel qu'il se répartit par catégorie d'établissements. Les données montrent que l'augmentation d'effectifs n'a pas été la même partout. Elle a été minime dans les centres hospitaliers de soins de longue durée (CHSLD) et les centres de services sociaux (CSS), dont certaines responsabilités ont été attribuées à d'autres établissements. Dans les centres locaux de services communautaires (CLSC), l'augmentation a été la plus forte (+301,1\%), dans les centres d'accueil d'hébergement (CAH) (+73,1\%), dans les centres d'accueil de réadaptation (CAR) $(+37,7 \%)$ et dans les hôpitaux de soins de courte durée $(+29,6 \%)$ dont le nombre de lits a pourtant baissé au cours de la période. Ces changements traduisent un réajustement des services aux besoins d'une population qui connaît un vieillissement démographique rapide entraînant une transformation du tableau épidémiologique. Les besoins en hébergement, en réadaptation, en soins à domicile sont plus grands, et même les hôpitaux de soins aigus doivent s'adapter à une clientèle "plus lourde ", affligée de problèmes plus difficiles à traiter et souvent multiples.

L'augmentation d'effectifs depuis 1980 est surtout attribuable à l'augmentation du nombre de postes à temps partiel (tableau 3). Au début de la période, beaucoup de postes à temps complet ont été transformés en postes à temps partiel, en réponse aux contraintes imposées par la récession économique. La tendance s'est maintenue jusqu'en 199 1, à la différence qu'à partir du milieu de la décennie, sous la pression syndicale, les directions d'établissement ont créé des postes à temps partiel régulier, plutôt qu'occasionnels. En ce sens, la proportion d'emplois précaires a diminué au profit d'emplois stables, quoique à temps partiel. 


\section{Tableau 2}

Personnel clinique, en équivalents en temps plein (ETP) Services de santé et services sociaux, Province de Québec, 1980, 1984, 1991

$\underline{\text { Retour à la table des matières }}$

\begin{tabular}{|c|c|c|c|c|c|}
\hline & \multicolumn{3}{|c|}{ Nombre de postes ETP } & \multicolumn{2}{|c|}{$\begin{array}{l}\text { Accroissement } \\
1980=100\end{array}$} \\
\hline & 1980 & 1984 & 1991 & 1984 & 1991 \\
\hline Centres d'accueil d'hébergement & 5191 & 6541 & 8985 & 126 & 173 \\
\hline Centres d'accueil de réadaptation & 6823 & 7503 & 9395 & 110 & 138 \\
\hline $\begin{array}{l}\text { Centres hospitaliers de soins de courte } \\
\text { durée }\end{array}$ & 45531 & 45753 & 59009 & 100 & 130 \\
\hline Centres hospitaliers psychiatriques & 7409 & 7031 & 8484 & 45 & 115 \\
\hline $\begin{array}{l}\text { Centres hospitaliers de soins de longue } \\
\text { durée }\end{array}$ & 6197 & 6660 & 6332 & 107 & 102 \\
\hline $\begin{array}{l}\text { Centres locaux de services communau- } \\
\text { taires }\end{array}$ & 2644 & 4206 & 7963 & 159 & 301 \\
\hline $\begin{array}{l}\text { Conseils régionaux de la santé et des } \\
\text { services sociaux }\end{array}$ & 5 & 34 & 372 & 680 & 7440 \\
\hline Centres de services sociaux & 3862 & 3773 & 3889 & 98 & 101 \\
\hline Établissements privés & 3627 & 3082 & 4694 & 85 & 129 \\
\hline TOTAL & 81289 & 84583 & 109123 & 104 & 134 \\
\hline
\end{tabular}




\section{Tableau 3}

Nombre de postes, selon le statut d'emploi, personnel clinique des services de santé et des services sociaux, Province de Québec, 1980, 1984, 1991

$\underline{\text { Retour à la table des matières }}$

\begin{tabular}{lcccccc}
\hline & \multicolumn{2}{c}{1980} & \multicolumn{2}{c}{1984} & \multicolumn{2}{c}{1991} \\
\hline $\begin{array}{l}\text { Nombre de postes à } \\
\text { temps complet régulier }\end{array}$ & 65164 & $(49,4 \%)$ & 60169 & $(42,6 \%)$ & 69300 & $(43,5 \%)$ \\
$\begin{array}{l}\text { Nombre de postes à } \\
\text { temps partiel régulier }\end{array}$ & 21391 & $(16,2 \%)$ & 29031 & $(20,5 \%)$ & 45768 & $(28,7 \%)$ \\
$\begin{array}{l}\text { Nombre de postes à } \\
\text { temps partiel occasionnel }\end{array}$ & 45336 & $(34,4 \%)$ & 52155 & $(36,9 \%)$ & 44208 & $(27,8 \%)$ \\
TOTAL & 131891 & & 141355 & & 159276 & \\
\hline
\end{tabular}

Sources: G. Dussault et al. (1987), La main-d'oeuvre socio-sanitaire au Québec, Québec, Commission d'enquête sur les services de santé et les services sociaux, p. 234 et 357. Ministère de la Santé et des Services sociaux, Province de Québec (1992), Statistiques sur le personnel de la santé et des services sociaux, 1990-1991, Québec.

En 1990-1991, les emplois à temps partiel représentaient environ $56 \%$ de l'ensemble des postes et se répartissaient à peu près également entre postes à temps partiel régulier et occasionnel. Par contre, la précarité n'est pas également distribuée entre les catégories d'emploi ni entre les catégories d'établissements (MSSS, 1992, p. 59-60). Ainsi chez les professionnels $(70,9)$, chez les infirmières bachelières $(67,2)$, chez les techniciens $(62,1)$, le pourcentage d'heures travaillées à temps plein, l'indicateur le plus exact du niveau d'activité, est nettement supérieur à ce qu'il est chez les infirmières $(48,4)$ et chez les assistantstechniciens $(46,8)$, qui constituent la main-d'oeuvre la plus abondante et se prêtant le plus facilement à la substitution. Selon le type d'établissement, les pourcentages d'heures travaillées par du personnel à temps complet régulier varient de 41,2 dans les CAH à 80,5 dans les CSS ; dans les autres catégories, les pourcentages se situent entre 50\% et $60 \%$. Les heures travaillées par le personnel le plus précaire, celui 
qui travaille à temps partiel occasionnel, varient de 19\% dans les CHSLD à 26\% dans les CAR. Ces différences reflètent vraisemblablement des variations de pratiques administratives et de rapports avec les syndicats d'employés. Il n'est toutefois pas possible d'apprécier l'impact de l'importance plus ou moins grande du travail précaire sur la qualité des services.

\section{Les effectifs des corporations professionnelles (1978-1992)}

$\underline{\text { Retour à la table des matières }}$

Les membres des 22 professions du secteur sociosanitaire reconnues par le Code des professions du Québec constituent le bassin de producteurs potentiels de services correspondant aux champs d'activité de leur occupation. Dans le cas des professions dites "d'exercice exclusif » l'appartenance à la corporation professionnelle est une obligation pour quiconque veut exercer. Il s'ensuit que le nombre de membres de ces corporations représente le bassin maximal réel de producteurs. En réalité, toutefois, tous les membres en règle d'une corporation professionnelle d'exercice exclusif ne sont pas des producteurs de soins ou de services : certains n'exercent aucune activité professionnelle pour des raisons diverses (retraite, retour aux études, congés prolongés de toutes sortes), d'autres sont des enseignants, des administrateurs, des chercheurs à temps plein. Parmi ceux qui produisent des services, le niveau d'activité peut varier beaucoup, de sorte que pour connaître la véritable production il faudrait savoir ce que font ces professionnels. À notre connaissance, le seul cas de professionnels dont on connaît un tant soit peu le niveau d'activité est celui des médecins, dont il sera question plus loin.

L'exercice des activités correspondant aux champs réglementés par les corporations dites «de titre réservé » n'est pas limité aux seuls 
membres de ces corporations. On peut, par exemple, exercer des activités d'hygiéniste dentaire sans être membre de la Corporation professionnelle des hygiénistes dentaires; on ne peut toutefois utiliser le titre d'« hygiéniste dentaire ». Il en résulte que les données sur les effectifs des corporations de cette catégorie ne représentent pas le bassin total des producteurs potentiels. La tendance, depuis l'adoption du Code des professions en 1973, a été, dans le réseau des affaires sociales, de recruter prioritairement des professionnels membres d'une corporation ; on peut ainsi estimer que les données des corporations dont les membres exercent majoritairement dans les services publics, reflètent mieux le bassin total, puisque pour travailler, il était avantageux d'être membre de la corporation. Si on ajoute ces limites à celles déjà mentionnées relativement à notre déficit d'informations sur l'activité réelle des professionnels, il faut traiter les données qui suivent avec précaution.

Le tableau 4 décrit l'évolution des effectifs des corporations professionnelles, en nombres absolus, de 1978 à1992. Ceux des corporations d'exercice exclusif ont augmenté, au cours de la période, cinq fois plus que la population du Québec (42,3\% et 8,6\% respectivement). Certaines occupations ont même connu un accroissement encore plus spectaculaire ; jusqu'à sept ou huit fois la croissance de la population (chiropraticiens, opticiens d'ordonnance). Il n'est pas possible de porter un jugement sur ce phénomène en ce qui a trait à l'opportunité de cette croissance : s'agit-il d'un rattrapage, ou d'un excès de production ? Il est difficile de répondre à cette question en l'absence de normes quant au nombre de professionnels de chaque catégorie qui serait théoriquement nécessaire pour répondre aux besoins. Du côté des professions de titre réservé, on observe une croissance de même envergure, mais plus inégalement répartie entre les occupations qui composent cette catégorie. Si on exclut de l'analyse les données sur les psychologues et sur les travailleurs sociaux, en raison de la proportion importante de membres qui ne travaillent pas directement dans le secteur sociosanitaire, il appert que sept occupations ont connu une croissance très élevée (de 10 à 32 fois plus rapide que la popula- 
tion) alors que trois autres avaient une croissance beaucoup plus modeste, tout en restant supérieure à l'accroissement démographique. C'est le cas des technologistes médicaux et des infirmières auxiliaires qui font face, chaque groupe à sa manière, à un processus de substitution. Dans le premier cas, la «menace » vient surtout de l'automatisation de certaines tâches d'analyse ; dans le second cas, le processus est plus complexe. D'un côté, les infirmières, au nom d'une approche des soins qui valorise une prise en charge plus totale, se substituent, dans le secteur des soins aigus, de plus en plus aux auxiliaires. De l'autre, les établissements de soins utilisent davantage des personnels moins spécialisés (préposés aux bénéficiaires) par mesure d'économie et pour profiter de la plus grande flexibilité que permet le recours à cette main-d'œuvre plus mobile. Notons qu'en plus, des politiques d'encouragement au recyclage pour passer au statut d'infirmière ont aussi contribué à l'attrition des effectifs d'infirmières auxiliaires. Quant aux augmentations impressionnantes des effectifs des autres occupations, elles sont probablement le fait à la fois d'une augmentation du nombre de professionnels, en réponse à une demande à la hausse, et d'un meilleur recrutement de la part des corporations. 
Tableau 4

Effectifs des corporations professionnelles du secteur de la santé et des services sociaux, Province de Québec, 1978,1984,1992

$\underline{\text { Retour à la table des matières }}$

\begin{tabular}{|c|c|c|c|c|c|}
\hline & \multicolumn{3}{|c|}{ Nombre de membres } & \multicolumn{2}{|c|}{$\begin{array}{c}\text { Accroissement } \\
1978=100\end{array}$} \\
\hline & 1978 & 1984 & 1992 & 1984 & 1992 \\
\hline \multicolumn{6}{|l|}{ Corporations d'exercice exclusif } \\
\hline 1) Audioprothésistes & 100 & 96 & 145 & 96 & 145 \\
\hline 2) Chiropraticiens & 479 & 579 & 775 & 121 & 162 \\
\hline 3) Dentistes & 2258 & 2742 & 3294 & 121 & 146 \\
\hline 4) Denturologistes & 629 & 844 & 909 & 134 & 145 \\
\hline 5) Infirmières & 45527 & 52956 & 63712 & 116 & 140 \\
\hline 6) Médecins & 11247 & 13304 & 16625 & 118 & 149 \\
\hline 7) Opticiens d'ordonnance & 396 & 469 & 959 & 118 & 242 \\
\hline 8) Optométristes & 710 & 866 & 1089 & 122 & 153 \\
\hline 9) Pharmaciens & 3144 & 3852 & 4860 & 123 & 156 \\
\hline 10) Podiatres & 127 & 117 & 98 & 92 & 77 \\
\hline 11) Techniciens en radiologie & 2514 & 2816 & 3386 & 112 & 135 \\
\hline Sous-total & 67131 & 78641 & 95852 & 117 & 142 \\
\hline \multicolumn{6}{|l|}{ Corporations de titre réservé } \\
\hline 1) Diététistes & 951 & 1219 & 1748 & 128 & 184 \\
\hline 2) Ergothérapeutes & 388 & 693 & 1393 & 179 & 359 \\
\hline 3) Hygiénistes dentaires & 609 & 1164 & 2284 & 191 & 375 \\
\hline 4) Infirmières auxiliaires & 16674 & 18848 & 19565 & 111 & 117 \\
\hline 5) Inhalothérapeutes & 897 & 1318 & 1725 & 147 & 192 \\
\hline 6) Orthophonistes, audiologistes & 241 & 445 & 665 & 185 & 276 \\
\hline 7) Physiothérapeutes & 905 & 1407 & 2427 & 155 & 268 \\
\hline 8) Psychologues & 1889 & 2947 & 5217 & 156 & 276 \\
\hline 9) Techniciens dentaires & 217 & 252 & 302 & 116 & 139 \\
\hline 10) Technologistes médicaux & 2039 & 1974 & 2342 & 97 & 115 \\
\hline 11) Travailleurs sociaux & 1803 & 1906 & 3055 & 106 & 169 \\
\hline Sous-total & 26613 & 32173 & 40723 & 131 & 166 \\
\hline TOTAL & 93744 & 110814 & 136575 & 121 & 149 \\
\hline
\end{tabular}

Sources : Rapports annuels des corporations professionnelles pour 1978, 1984. Rapport annuel de l'Office des professions pour 1992. 
Une analyse plus poussée de la production sanitaire exige plus d'informations que celles qui sont généralement disponibles. Nous présentons brièvement l'exemple des médecins, profession plus étudiée sous ce rapport que les autres, afin d'illustrer notre propos. Le tableau 5 réunit des informations qui mettent en évidence des changements, dans la composition du bassin de médecins, qui ont vraisemblablement des effets sur le type et le volume de soins et services produits. Ainsi, depuis 1972, la proportion de médecins spécialistes a diminué graduellement pour devenir équivalente à celle des omnipraticiens ; il en résulte que la production de services généraux a augmenté en proportion et que le coût total des services médicaux est probablement moins élevé qu'il ne l'aurait été si les spécialistes étaient restés plus nombreux. L'augmentation plus rapide du nombre de généralistes a été accompagnée d'une meilleure répartition régionale de leurs effectifs, ce qui a favorisé l'accès à leurs services. On observe aussi une féminisation graduelle de la profession médicale, ce qui a également un effet sur la production en raison des différences d'attitudes et de comportement entre médecins hommes et médecins femmes (Maheux et Dufort, 1990). Cela se traduit en particulier par une baisse graduelle du nombre d'heures consacrées aux soins : avec le temps, les comportements des hommes et des femmes ont tendance à se ressembler de plus en plus (Contandriopoulos et Fournier, 1992) pendant que les hommes réduisent graduellement leur niveau d'activité. Des études plus poussées seraient nécessaires pour expliquer ce phénomène et étudier l'influence de facteurs, comme la plus grande disponibilité de médecins, des changements de valeurs, d'attitudes face à l'exercice professionnel et au rôle de médecin. Enfin, les données du tableau 5 permettent d'observer le vieillissement rapide des médecins spécialistes. Il y avait en 1990 au moins huit spécialités cliniques ${ }^{8}$ dont plus du quart des membres avaient plus de 60 ans, âge auquel le niveau d'activité commence à baisser sensiblement (tableau 6). Au total, la

8 Chirurgie générale $(36,7)$, physiatrie $(30,9)$, médecine interne $(28,9)$, anesthésie-réanimation $(26,8)$, neuro-chirurgie $(26,7)$, obstétrique-gynécologie $(26,1)$, chirurgie cardiovasculaire et thoracique $(26,1)$, pneumologie $(25,0)$. 
proportion de spécialistes, toutes spécialités confondues, âgés de 60 ans et plus est passée de 16,4\% en 1980 à 23,2\% en 1990. Ces informations sont utiles à la prévision des besoins en effectifs nouveaux : elles ne sont toutefois pas toujours disponibles et même lorsqu'elles le sont, elles sont rarement utilisées, la planification de la main-d'œuvre sociosanitaire, à l'exception notable de la main-d'œuvre médicale, étant presque inexistante.

\section{Tableau 5}

Données sur les effectifs médicaux au Québec, 1972, 1978, 1984, 1990

Retour à la table des matières

\begin{tabular}{|c|c|c|c|c|c|c|c|c|}
\hline & \multicolumn{2}{|c|}{1972} & \multicolumn{2}{|c|}{1978} & \multicolumn{2}{|c|}{1984} & \multicolumn{2}{|c|}{1990} \\
\hline $\begin{array}{l}\text { 1. Nombre total de méde- } \\
\text { cins (1) (2) }\end{array}$ & 7498 & & 10113 & & 11973 & & 14907 & \\
\hline 2. Population par médecin & 813 & & 624 & & 542 & & 480 & \\
\hline $\begin{array}{l}\text { 3. Nombre d'omniprati- } \\
\text { ciens }\end{array}$ & 3160 & $(42,1 \%)$ & 4728 & $(46,8 \%)$ & 5885 & $(49,2 \%)$ & 6972 & $(49,5 \%)$ \\
\hline 3.1 \% Hommes & & 91,9 & & $(85,0$ & & $(77,3$ & & $(70,4$ \\
\hline \% Femmes & & 7,1 & & $(15,0$ & & $(22,7$ & & $(29,6$ \\
\hline $\begin{array}{l}3.3 \% \text { Âgés de } 60 \text { ans } \\
\text { et plus }\end{array}$ & & 14,7 & & $(11,0$ & & $(12,2$ & & $(12,5$ \\
\hline $\begin{array}{l}\text { Nombre moyen } \\
\text { d'heures consa- } \\
\text { crées aux soins } \\
\text { des patients par } \\
\text { semaine }\end{array}$ & & n.d. & & 43,5 & & $(42,3$ & & $(39,4$ \\
\hline 4. Nombre de spécialistes & 4338 & $(57,9 \%)$ & 5385 & $(53,2 \%)$ & 6088 & $(50,8 \%)$ & 7125 & $(50,5 \%)$ \\
\hline $4.1 \%$ Hommes & & 94,9 & & $(92,8$ & & $(89,4$ & & $(84,7$ \\
\hline 4.2 \% Femmes & & 5,1 & & 7,2 & & $(10,6$ & & $(15,3$ \\
\hline $\begin{array}{l}4.3 \% \text { Âgés de } 60 \text { ans } \\
\text { et plus }\end{array}$ & & 12,8 & & $(14,4$ & & $(19,6$ & & $(23,3$ \\
\hline 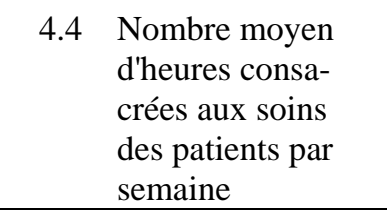 & & n.d. & & 41,2 & & $(40,6$ & & $(39,8$ \\
\hline
\end{tabular}

(1) Nombre de médecins titulaires d'un permis d'exercer dont le principal lieu de pratique déclaré est le Québec.

(2) Données recueillies par questionnaire au moment du paiement de la cotisation annuelle. Source : A.P. Contandriopoulos et al. (1992), Les effectifs médicaux au Québec. Situation de 1972 à 1990 et projections pour 1995, Montréal, Corporation professionnelle des médecins du Québec. 


\section{Tableau 6}

Âge moyen et proportion de médecins âgés de 60 ans et plus selon la spécialité, au Québec, 1990

$\underline{\text { Retour à la table des matières }}$

\begin{tabular}{|c|c|c|}
\hline \multirow{2}{*}{ Spécialité } & Age moyen & $\begin{array}{l}\text { \% des médecins âgés } \\
\text { de } 60 \text { ans et plus }\end{array}$ \\
\hline & 1990 & 1990 \\
\hline Anatomo-pathologie & 52,3 & 31,7 \\
\hline Anesthésie-réanimation & 49,8 & 26,8 \\
\hline Biochimie médicale & 49,7 & 17,1 \\
\hline Cardiologie & 48,9 & 22,2 \\
\hline Chirurgie cardiovasculaire et thoracique & 50,2 & 26,1 \\
\hline Chirurgie générale & 54,4 & 36,7 \\
\hline Chirurgie orthopédique & 49,5 & 20,1 \\
\hline Chirurgie plastique & 47,8 & 17,9 \\
\hline Dermatologie & 46,0 & 10,7 \\
\hline Endocrinologie & 49,7 & 16,1 \\
\hline Gastro-entérologie & 47,5 & 16,4 \\
\hline Gériatrie & 41,8 & 15,4 \\
\hline Hématologie & 48,0 & 17,0 \\
\hline Immunologie clinique et Allergie & 47,7 & 13,2 \\
\hline Médecine interne & 49,9 & 28,9 \\
\hline Médecine nucléaire & 44,9 & 19,7 \\
\hline Microbiologie médicale et Infectiologie & 46,6 & 15,6 \\
\hline Néphrologie & 46,8 & 10,1 \\
\hline Neurochirurgie & 51,5 & 26,7 \\
\hline Neurologie & 47,2 & 12,7 \\
\hline Obstétrique-gynécologie & 50,3 & 26,1 \\
\hline Ophtalmologie & 49,4 & 21,9 \\
\hline Oto-rhino-laryngologie & 51,3 & 24,3 \\
\hline Pédiatrie & 47,9 & 20,1 \\
\hline Physiatrie & 51,6 & 30,9 \\
\hline Pneumologie & 48,6 & 25,0 \\
\hline Psychiatrie & 48,3 & 19,7 \\
\hline Radiologie diagnostique & 50,1 & 24,1 \\
\hline Radio-oncologie & 47,9 & 15,4 \\
\hline Rhumatologie & 46,4 & 15,5 \\
\hline Santé communautaire & 51,1 & 30,3 \\
\hline Urologie & 49,7 & 18,2 \\
\hline TOTAL & 49,5 & 23,2 \\
\hline
\end{tabular}


Source : A. P. Contandriopoulos et al. (1992), Les effectifs médicaux au Québec. Situation de 1972 à 1990 et projections pour 1995, Montréal, Corporation professionnelle des médecins du Québec, p. 48.

\section{Les producteurs non reconnus}

$\underline{\text { Retour à la table des matières }}$

Il importe de dire quelques mots des producteurs non reconnus parce que depuis les années 1970, ils ont été nombreux à arriver sur le marché des soins ; ils y offrent des services différents, complémentaires, tantôt alternatifs aux services officiels. On les retrouve essentiellement dans les domaines de la médecine chinoise, des manipulations (massage thérapeutique, ostéopathie, orthothérapie), de l'homéopathie et des "médications naturelles » (phytothérapie, naturopathie), des approches énergétiques ${ }^{9}$ (polarité, réflexologie) et des psychothérapies. Certains groupes s'affichent ouvertement, ont formé des associations, créé des écoles, et revendiquent une reconnaissance officielle (Dussault et al., 1987 ; Office des professions du Québec, 1992). Certains, comme les acupuncteurs, sont déjà l'objet d'une réglementation et d'une reconnaissance officielle. La manière dont on a réglementé l'exercice de l'acupuncture n'a toutefois pas satisfait tous les acupuncteurs ${ }^{10}$, dont une bonne partie estiment avoir été mis en tutelle par les médecins et refusent de s'enregistrer (Voyer, 1989).

9 Approches qui ont « pour but l'harmonisation et la circulation optimale de l'énergie vitale dans le corps humain afin de le maintenir en santé ou de stimuler ses capacités naturelles d'auto-guérison et ses fonctions organiques » (Office des professions du Québec, 1992, p. 4).

10 Pour exercer légalement, les acupuncteurs sont tenus de réussir un examen de la Corporation professionnelle des médecins du Québec et de s'inscrire auprès de celle-ci. 
On sait peu de choses au sujet de cette catégorie de producteurs; cela tient justement au fait de leur marginalité et du caractère plus ou moins clandestin de leur exercice. Une étude effectuée en 1987 (Dussault et al., 1987) estimait à 7000 approximativement le bassin de ces producteurs, à partir d'un recensement des effectifs des associations connues et en faisant l'hypothèse qu'elles ne représentaient qu'une fraction du nombre réel de producteurs. Cette étude notait que, parmi les producteurs potentiels, un grand nombre n'offrait pas de services contre rémunération; par exemple, il y avait à l'époque plus de 2000 " phytothérapeutes » qui en réalité étaient pour la plupart des personnes qui avaient suivi une formation à des fins personnelles et qui limitaient leurs activités à l'autotraitement ou à leur cercle de parents et d'amis. Il est probable qu'on retrouve des cas semblables dans plusieurs autres domaines notamment ceux du massage et des approches énergétiques, de sorte que le nombre réel de producteurs non reconnus qui offrent leurs services contre rémunération sur une base régulière était plutôt de l'ordre de la moitié du bassin total estimé.

Une étude subséquente (Rousseau et al., 1991) a tenté de caractériser cette population par le biais d'une enquête auprès de producteurs qui annonçaient leurs services dans divers médias. Au moment de l'enquête, les auteurs ont répertorié 954 annonceurs (individus et groupes) et en ont joint 319 qui présentaient les caractéristiques suivantes : ils avaient tendance à se concentrer dans les zones urbaines, se distribuaient à peu près également entre hommes et femmes ; l'âge moyen était de 40,1 ans et les trois quarts exerçaient leur métier depuis moins de 10 ans. En excluant quelques cas marginaux qui déclaraient des revenus annuels de plus de $100000 \$$, le revenu annuel moyen était de l'ordre de $22000 \$$ et montait à $30000 \$$ si on ne considérait que ceux dont la totalité des revenus provenait de la prestation de ces services. Ces données sont intéressantes du fait qu'elles sont les premières véritables données d'enquête ; elles sont toutefois difficiles, sinon impossibles, à interpréter, puisqu'on ne connaît pas la population dans son ensemble et qu'on ne peut, en conséquence, estimer la représentativité des informations recueillies. 
Enfin, plus récemment, l'Office des professions du Québec (1992) a étudié les requêtes en incorporation de plusieurs associations de producteurs non reconnus. En recoupant les données contenues dans son Avis sur l'opportunité de créer des corporations professionnelles dans les domaines où exercent ces producteurs, on peut estimer que le bassin de ces producteurs est vraisemblablement de l'ordre de 6000 à 7 000, sans égard au niveau d'activité. L'Office des professions estimait qu'il y avait près de 2000 praticiens de thérapies manuelles (regroupés en 12 associations), 400 dans l'homéopathie et les médications naturelles, auxquels s'ajoute un réseau de 2000 vendeurs de produits de phytothérapie et environ 300 praticiens des approches énergétiques. À ce total de 2700 (4 700 incluant les «vendeurs »), on peut ajouter environ 400 acupuncteurs qui continuent à exercer leur travail sans être inscrits au Registre des acupuncteurs de la Corporation professionnelle des médecins et les praticiens du secteur des psychothérapies qui n'ont pas été comptabilisés dans l'Avis de l'Office, mais dont le nombre a été estimé par son service de recherche à environ 4 000 (Doyle, 1993).

En somme, il reste difficile de dire quel est le bassin réel de producteurs non reconnus, quel est leur volume de production et quelle est l'importance relative de cette production dans le système de soins.

\section{Tendances de l'évolution des effectifs sociosanitaires}

$\underline{\text { Retour à la table des matières }}$

Trois phénomènes observés dans l'évolution des effectifs nous semblent porteurs d'impacts importants pour l'avenir prochain du système de soins. Il y a la croissance continue des effectifs, deux fois plus importante que celle de la population ; et surtout le fait que cette croissance est principalement due à la croissance des personnels plus 
qualifiés (professionnels, infirmières, techniciens). Un second phénomène est celui de l'accroissement d'une certaine précarité de l'emploi, qui prend la forme de l'augmentation des emplois à temps partiel réguliers et occasionnels. Le troisième phénomène, plus difficile à documenter, est celui de l'apparition d'un marché de soins parallèle au marché officiel : le phénomène intéressant est que ce marché semble être là pour durer et être plus que le résultat d'un engouement passager.

Il est difficile de juger si l'augmentation des effectifs, en proportion plus grande que l'augmentation de population, correspond à des besoins réels. D'une part, on ne sait pas si elle entraîne une augmentation correspondante du volume de production de soins et services, faute de données sur la productivité. D'autre part, notre connaissance des besoins reste très imparfaite, de même que celle de la capacité qu'ont les services produits d'y répondre adéquatement. Cela dit, il est raisonnable de prévoir que le type de croissance observé exerce une forte pression à la hausse sur les coûts totaux (publics, privés) des soins. En effet, la croissance est plus rapide dans les catégories de producteurs les plus qualifiés, qui coûtent le plus cher à employer et qui ont plus de marge décisionnelle pour stimuler la demande et l'utilisation des services. Les professionnels, dont le nombre a doublé depuis la fin des années 1970, sont ceux qui posent les diagnostics et formulent les Prescriptions qui induisent l'utilisation. Ils ont aujourd'hui accès à des outils de diagnostic plus efficaces et sont plus en mesure d'établir ce qui, de leur point de vue, constitue un besoin. Étant plus nombreux et mieux outillés, on peut supposer que le volume de diagnostics va augmenter et, à moins de contraintes nouvelles à l'utilisation, on observera une croissance du recours aux services de santé.

On peut également penser que la concurrence accrue entre les professionnels, qui sont plus nombreux pour une clientèle proportionnellement moindre, va décourager le recours à la substitution de personnel comme moyen de diminuer les coûts des services. La législation professionnelle en vigueur, de même que les contrats de travail liant 
les professionnels aux établissements ne favorisent pas la substitution, bien au contraire (Commission d'enquête sur la santé et les services sociaux, 1988). On risque donc d'observer une croissance de la demande induite pour les services les plus coûteux, ce qui ne peut se traduire que par une forte croissance des coûts si cette demande est satisfaite. Cette croissance des coûts peut en même temps s'accompagner d'une réduction de l'accès à certains services. En diminuant le personnel moins qualifié parce que les plus qualifiés coûtent trop cher, au lieu de déléguer davantage de tâches à ce personnel, on risque de réduire l'accès aux services de base et aux services de soutien.

Ensuite, le recours accru aux options thérapeutiques offertes sur le marché parallèle n'est pas un substitut à l'utilisation des services « officiels ». S'il l'était, comme les services du marché parallèle sont moins coûteux, du fait de la non-utilisation d'équipements et de médicaments chers, il en résulterait une diminution des coûts. Il semble toutefois que ces services sont souvent utilisés en complémentarité avec les services traditionnels et que leurs coûts représentent un ajout aux coûts totaux des services, car la consommation de services assurés n'en diminue pas pour autant.

Les enjeux soulevés par l'importance accrue de la précarité d'emploi sont de divers ordres. Pour les employés, il y a évidemment une question d'insécurité, de difficulté d'accès à un travail «normal » qui offre la possibilité de faire carrière. Pour les plus qualifiés, la difficulté d'accéder à un emploi à temps plein entraîne le risque de la diminution des capacités techniques. Chez les infirmières, cette difficulté est souvent citée comme cause importante des problèmes de recrutement (MSSS, 1987). Pour la clientèle, la précarité peut signifier une qualité de services potentiellement moindre. Les employés qui travaillent moins ou combinent plus d'un poste, n'ont pas les mêmes possibilités de développer leurs habiletés que ceux qui ont un emploi stable à temps complet. Surtout la continuité des services est plus difficilement assurée lorsque le roulement de personnel est élevé. L'absence de continuité peut être une source de confusion pour la clientèle 
et une source d'erreurs diverses occasionnées par la manipulation des dossiers par plusieurs personnes et par des problèmes de transmission d'informations.

L'existence durable d'un marché parallèle de soins soulève l'enjeu de sa réglementation et éventuellement de son intégration au système officiel. La question de la réglementation est d'abord celle de la reconnaissance de la légitimité de cette production au plan juridique. Pour les producteurs non reconnus, il en va de leur capacité même à offrir légalement leurs services. Actuellement, ils le font dans une relative clandestinité : les corporations professionnelles, responsables des poursuites pour exercice illégal, et le ministère de la Santé et des Services sociaux ont adopté une attitude de laissez-faire relatif. Même la Corporation des médecins, qui tient un discours ouvertement antimédecines alternatives, n'a jamais poursuivi, pour exercice illégal de la médecine, plus de 100 personnes par année ; il est vrai que cela suffit amplement à semer la crainte dans les rangs des praticiens non reconnus. Quant au Ministère sa position a été longtemps d'observer et d'étudier la situation sans prendre de position ferme.

Pour l'État, les enjeux du marché parallèle sont ceux de la protection du public et de l'intégration éventuelle de ces services dans le système de soins (Dussault, 1988). Dans le premier cas, il s'agit de veiller à ce que la population ne soit pas exposée à des dangers qui pourraient être évités en empêchant que des charlatans offrent leurs services. Dans le second cas, les implications d'une reconnaissance des nouveaux producteurs peuvent être lourdes aux plans politique et économique. Les reconnaître signifie, actuellement du moins, mécontenter les producteurs reconnus qui ne manqueront pas de s'opposer à ce que " leur marché » soit ouvert à de nouveaux venus, surtout qu'il est devenu clair que les ressources disponibles n'augmenteront pas. Ne pas les reconnaître, c'est mécontenter une partie de la population qui estime avoir le droit de choisir les modalités thérapeutiques qu'elle préfère. Quant aux implications économiques, elles peuvent varier selon le type de reconnaissance concédé. L'État peut, à un extrême, recon- 
naître les nouveaux producteurs et ne rien faire pour favoriser l'accès à leurs services. Il y a des précédents comme dans le cas des chiropraticiens. Après avoir exercé dans la clandestinité pendant environ 50 ans, ils ont été reconnus par le Code des professions en 1973 ; par contre, rien n'a été fait pour les intégrer au réseau public de services de santé, ni, jusqu'à récemment ${ }^{11}$, pour leur donner accès au réseau d'éducation. À l'autre extrême, la reconnaissance peut s'accompagner d'une intégration dans les services publics et par la mise en place de programmes de formation. Selon l'option préférée, les coûts vont de presque rien à des sommes importantes.

\section{CONCLUSION}

$\underline{\text { Retour à la table des matières }}$

Le système de soins parfait serait celui qui parviendrait à satisfaire efficacement les véritables besoins, c'est-à-dire à leur apporter une réponse adéquate, au bon moment, au bon endroit, avec les moyens appropriés, utilisés de la manière la plus efficiente. Cet idéal de soins efficaces, de haute qualité, accessibles et efficients exigerait qu'on utilise toutes les ressources de façon optimale. C'est une utopie, au sens d'un objectif qu'on sait impossible à atteindre mais qu'il vaut la peine de continuer à viser.

Compte tenu de la nature même des services sociosanitaires, qui sont par définition des services donnés par des personnes à d'autres personnes, la « ressource » la plus stratégique reste le producteur de services. Les résultats du système de soins dépendent essentiellement des producteurs et de leurs comportements, qui eux-mêmes sont influencés par plusieurs groupes de facteurs liés aux ressources à leur

11 Les chiropraticiens devaient auparavant aller suivre leur formation en Ontario ou aux Etats-Unis. Un programme de formation est offert par l'Université du Québec à Trois-Rivières depuis septembre 1993. 
disposition, au contexte organisationnel dans lequel ils travaillent, aux pressions dont ils sont l'objet par les utilisateurs. Dans ce chapitre, nous nous sommes intéressé exclusivement au bassin de producteurs : nous avons avancé qu'il est important d'en connaître la composition et la manière dont il évolue, pour être davantage en mesure de prédire les tendances de la production. Nous avons dû constater que l'information disponible est insuffisante pour tracer un portrait valide des producteurs et que nous savons peu de choses au sujet de leurs comportements véritables. La chose étonne un peu compte tenu des enjeux économiques et sociosanitaires (volume et qualité des services) associés à l'évolution de cette main-d'œuvre. Peu d'efforts systématiques ont été consacrés à en connaître les caractéristiques, à essayer de mieux comprendre ses comportements. Il en va de même pour la connaissance des besoins auxquels ces producteurs disent répondre : ce n'est que depuis quelques années qu'on accepte de dépenser une fraction minime des sommes consacrées aux services sociosanitaires, pour tenter, par des enquêtes épidémiologiques rigoureuses, de connaître les états de santé et la distribution des problèmes dans la population. Jusque-là, on s'est contenté d'essayer de répondre à la demande telle qu'elle s'exprimait ou telle que les professionnels la concevaient.

Il ne suffirait pas toutefois de mieux connaître les producteurs et leurs comportements pour parvenir à mettre en place une planification « rationnelle » de l'utilisation de la main-d'œuvre sociosanitaire. La division et l'organisation du travail sont négociées plus qu'elles ne sont dictées et il serait illusoire de penser que pour les rendre plus efficaces et efficientes, il suffirait d'avoir accès à de meilleures informations. En plaidant pour une meilleure connaissance des producteurs de services, nous ne pensons aucunement que l'information va réduire la nature sociopolitique du processus de division et d'organisation du marché des soins : ce processus restera un processus de négociation, mais au moins, les données sur lesquelles s'appuient les arguments des uns et des autres refléteront plus adéquatement les faits. Dans la mesure où on attend véritablement du système de soins qu'il contribue 
d'abord et avant tout au mieux-être de la population, cette proposition devrait être vue comme raisonnable.

\section{RÉFÉRENCES}

$\underline{\text { Retour à la table des matières }}$

Bardy, J.D. (1988), « Analyse socio-politique de la situation des sages-femmes au Québec », Mémoire de maîtrise en administration de la santé, Université de Montréal.

Commission d'enquête sur les services de santé et les services sociaux (1988), Rapport, Québec, Ministère de la Santé et des Services sociaux.

Contandriopoulos, A.-P., et al. (1992), Les effectifs médicaux au Québec. Situation de 1972 à 1990 et projections pour 1995, Montréal, Corporation des médecins du Québec.

Doyle, Y. (1993), « La réglementation du secteur des psychothérapies », conférence au 1er congrès de l'Association des psychologues du Québec, Québec, 30 janvier.

Dumais, A., et J. Lévesque (1986), L'auto-santé des individus et des groupes au Québec, Québec, Institut québécois de recherche sur la culture. [Texte disponible dans Les Classiques des sciences sociales. JMT.]

Dussault, G. (1988), « Les enjeux de la reconnaissance des médecines parallèles », Artère, vol. 6, no 3, p. 11-12. 
Dussault, G., et al. (1987), La main-d'œuvre socio-sanitaire au Québec, Québec, Commission d'enquête sur la santé et les services sociaux.

Eisenberg, J. (1985), "The State of Research about Physicians' Practice Patterns », Medical Care, vol. 23, no 5, p. 461-483.

Émond, A., et Louise Guyon (1988), Et la santé, ça va ? Rapport de l'Enquête Santé Québec, Gouvernement du Québec, Ministère de la Santé et des Services sociaux.

Maheux, B., et F. Dufort (1990), «Female Medical Practitionners : More Preventive and Patient Oriented ? ", Medical Care, vol. 28, no 1, p. 87-92.

Ministère de la Santé et des Services sociaux, Province de Québec (1987), Rapport du comité d'étude sur la main-d'œuvre en soins infirmiers, Québec, février.

Ministère de la Santé et des Services sociaux, Province de Québec (1992), Statistiques sur le personnel de la santé et des services sociaux, 1990-1991, Québec.

Office des professions du Québec (1992), Avis au ministre responsable de l'application des lois professionnelles sur l'opportunité de constituer une corporation professionnelle dans le domaine des médecines douces, Québec, avril.

Régie de l'assurance-maladie du Québec (RAMQ) (1992), Statistiques annuelles 1991, Québec, Service des communications de la Régie de l'assurance-maladie du Québec.

Rousseau, N., F. Saillant et D. Desjardins (1991), «Profil sociodémographique des thérapeutes holistes au Québec ", Revue canadienne de santé publique, vol. 82, septembre-octobre, p. 335-340. 
Voyer, B. (1990), « Coexistence structurée de systèmes médicaux. Le cas de la médecine et de l'acupuncture au Québec », Mémoire de maîtrise en science politique, Université de Montréal.

Fin du texte 